\title{
Prefrontal cortex and hippocampus subserve different components of working memory in rats
}

\author{
Taejib Yoon, ${ }^{1}$ Jeffrey Okada, ${ }^{1}$ Min W. Jung, ${ }^{2}$ and Jeansok J. Kimm 1,3,4 \\ ${ }^{1}$ Department of Psychology, University of Washington, Seattle, Washington 98195-1525, USA; ${ }^{2}$ Neuroscience Laboratory, \\ Institute for Medical Sciences, Ajou University School of Medicine, Suwon 443-721, Korea; ${ }^{3}$ Program in Neurobiology \& Behavior, \\ University of Washington, Seattle, Washington 98195-1525, USA
}

\begin{abstract}
Both the medial prefrontal cortex (mPFC) and hippocampus are implicated in working memory tasks in rodents. Specifically, it has been hypothesized that the mPFC is primarily engaged in the temporary storage and processing of information lasting from a subsecond to several seconds, while the hippocampal function becomes more critical as the working memory demand extends into longer temporal scales. Although these structures may be engaged in a temporally separable manner, the extent of their contributions in the "informational content" of working memory remains unclear. To investigate this issue, the $\mathrm{mPFC}$ and dorsal hippocampus ( $\mathrm{dHPC}$ ) were temporarily inactivated via targeted infusions of the $\mathrm{GABA}_{\mathrm{A}}$ receptor agonist muscimol in rats prior to their performance on a delayed alternation task (DAT), employing an automated figure-eight maze that required the animals to make alternating arm choice responses after 3-, 30-, and 60-sec delays for water reward. We report that inactivation of either the mPFC or dHPC significantly reduced DAT at all delay intervals tested. However, there were key qualitative differences in the behavioral effects. Specifically, mPFC inactivation selectively impaired working memory (i.e., arm choice accuracy) without altering reference memory (i.e., the maze task rule) and arm choice response latencies. In contrast, dHPC inactivation increased both reference memory errors and arm choice response latencies. Moreover, dHPC, but not mPFC, inactivation increased the incidence of successive working memory errors. These results suggest that while both the mPFC and hippocampus are necessarily involved in DAT, they seem to process different informational components associated with the memory task.
\end{abstract}

Working memory is generally defined as cognitive entities (or "central executive" mechanisms) relating to temporary storage and operation of information in both humans and animals (Baddeley and Hitch 1974; Goldman-Rakic 1996; Fuster 2001; Dudchenko 2004). The memory may be about simple sensory stimulus, relatively complex objects, or spatial location (Olton et al. 1979; Delatour and Gisquet-Verrier 1996; Floresco et al. 1997; Hampson et al. 1999). Evidence from primate studies originally implicated the prefrontal cortex (PFC) as being crucial for working memory (Baddeley 1986; Goldman-Rakic 1987; Miller et al. 1991). For instance, damage to the PFC produces impairments in various working memory tasks in humans and non-human primates (Kolb 1990; Fuster 1997; Stuss and Alexander 2000). Additionally, recording and brain imaging studies found neural activity correlates of working memory, i.e., increased PFC activity during the delay period (Fuster and Alexander 1971; Kubota and Niki 1971; Funahashi et al. 1989).

In rodents, a delayed alternation task (DAT; employing T, radial-arm, and figure-eight mazes) has been widely used to further investigate the PFC-working memory hypothesis (Murphy et al. 1996; Zahrt et al. 1997; Baeg et al. 2003, 2007; Schoenbaum et al. 2003; Birnbaum et al. 2004; Clinton et al. 2006). In this task, the animal is typically cued (via a discrete or spatial stimulus) to make a choice response to obtain a reward, but is prevented from responding until after some delay period (or working memory demand) has been imposed. While the PFC is critically involved, the hippocampus has also been implicated in working memory tasks, particularly with long delays (Lee and Kesner 2003). Consistent with this view are findings from delayed (non)matching-

\footnotetext{
${ }^{4}$ Corresponding author.
}

E-mail jeansokk@u.washington.edu; fax (206) 685-3157.

Article is online at http://www.learnmem.org/cgi/doi/10.1101//m.850808. to-sample tasks, where hippocampal lesions produce mild/no performance deficits at short delays but induce pronounced disruptions as the delay is extended (Scoville and Milner 1957; Jarrard 1993; Eichenbaum 2000; Maruki et al. 2001). Other studies, however, have shown that the hippocampus is important even for short-delay working memory tasks (Kesner et al. 1996; Hampson et al. 1999).

Anatomically, the PFC is directly connected to the ventral hippocampus and indirectly connected to the dorsal hippocampus via the thalamus (Ferino et al. 1987; Laroche et al. 2000; Thierry et al. 2000). Although there are longitudinal connections between the ventral-dorsal poles of the hippocampus (Amaral and Witter 1995), the lack of monosynaptic projections between the PFC and the dorsal hippocampus, a region implicated in spatial information processing (Moser et al. 1993), suggests that perhaps they belong to separate and parallel memory systems participating in spatial working memory tasks. If the PFC and the hippocampus are indeed engaged in processing similar information but in different temporal domains, then damage to either structure should produce relatively time-specific deficits in working memory performance. Alternatively, if the PFC and the hippocampus contribute to qualitatively different aspects of the memory task (but overlap in temporal domain), damaging either structure should similarly disrupt working memory performance. Thus, the present study sought to further explicate the roles of the PFC and hippocampus in working memory by employing a computer vision-based figure-eight maze (Fig. 1; Pedigo et al. 2006). In brief, this maze provides automated animal tracking, working memory delays, and reward delivery. Thus, the maze does not require experimenter-subject interaction during shaping, training, and testing (Fig. 2). The maze automation also enables examination of a number of behavioral variables that have not been measured in previous studies. We report that inactiva- 


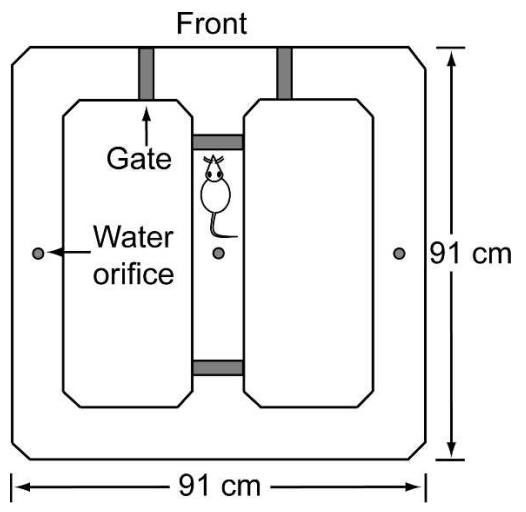

Figure 1. Schema of the automated figure-eight maze. The four gates are raised and lowered (from the maze surface) and water is delivered (at three reward locations) under computer control.

tion of either the PFC or the hippocampus profoundly impairs working memory performance, but the impairments are produced by interrupting different informational contents associated with DAT rather than differentially affecting delay times.

\section{Results}

\section{Histology}

Ten animals had guide cannulae bilaterally placed both within the borders of the mPFC (prelimbic and infralimbic areas; Fig. 3A) and dHPC (Fig. 3B). Because two rats had cannulae incorrectly placed in the mPFC (but correctly placed in the dHPC) and because three other rats had inoperative guide cannulae only in the $\mathrm{dHPC}$, the results from these animals were included for the dHPC and $\mathrm{MPFC}$ data analyses, respectively.

\section{Behavior}

Microinfusions of muscimol into either the mPFC (MUSC-mPFC) or dHPC (MUSC-dHPC) significantly impaired the performance on the DAT (Fig. 4). For mPFC infusion data, ANOVA with repeated measures on two factors (cf. Winer et al. 1991), with drug (ACSF and MUSC) and delay (3-, 30-, and 60-sec) as repeated measures, revealed significant main effects of drug $\left(F_{(1,12)}=123.38, P<0.001\right)$ and delay $\left(F_{(2,24)}=12.38, P<0.001\right)$, but not significant drug $\times$ delay interaction $\left(F_{(2,24)}=1.87\right.$, $P=0.18)$. For dHPC infusion data, there was a significant main effect of drug $\left(F_{(1,11)}=155.04, P<0.001\right)$, but neither main effect of delay $\left(F_{(2,22)}=0.57, P=0.57\right)$ nor drug $\times$ delay interaction $\left(F_{(2,22)}=0.52, P=0.60\right)$ were reliable.

A novel dependent measure examined on the maze was the "choice reaction time" (CRT) of animals making an arm choice response (left/right turn). The CRT was quantified as the latency from the start of each trial (when the front and side gates are in the DOWN position) to the completion of arm choice response (when the side gates are in the UP position) (Fig. 5A). First, we compared CRT between the first session of 40 trials (following the completion of the shaping procedure) and the session when animals reached the criterion ( $\geq 75 \%$ correct trials) on the DAT (Fig. 5B). A paired $t$-test revealed that animals made significantly quicker arm choice responses during the criterion session compared with those during the first session $\left(t_{(7)}=4.34, P=0.003\right)$, indicating improvement in the performance on the DAT. The movement trajectory map from a representative animal illustrates reduced variability in the performance of the DAT across testing sessions (Fig. 5C). Further analysis revealed that during the first 20-trial testing sessions (Day 1; no infusion), the CRT for correct trials was significantly shorter than the CRT for incorrect trials (Fig. 6A). An ANOVA with choice (correct vs. incorrect trials) as the between-subjects factor and delay (3-, 30-, and 60-sec) as the repeated measure revealed significant main effects of choice $\left(F_{(1,46)}=13.53, P=0.001\right)$, delay $\left(F_{(2,92)}=5.37, P=0.006\right)$, and a significant choice $\times$ delay interaction $\left(F_{(2,92)}=3.68\right.$, $P=0.03)$. However, the difference in CRT between correct and incorrect trials diminished during the sessions with muscimol infusions (Fig. 6B,C). For the MUSC-mPFC condition (Fig. 6B), there was a significant main effect of delay $\left(F_{(2,48)}=5.47\right.$, $P=0.007)$, but neither main effect of choice $\left(F_{(1,24)}=0.11\right.$, $P=0.75)$ nor delay $\times$ choice interaction $\left(F_{(2,48)}=1.17, P=0.32\right)$ were reliable. Similarly, for the MUSC-dHPC condition (Fig. 6C), there was a significant main effect of delay $\left(F_{(2,44)}=10.83\right.$, $P<0.001)$, but neither main effect of choice $\left(F_{(1,22)}=0.09\right.$, $P=0.77)$ nor delay $\times$ choice interaction $\left(F_{(2,44)}=0.005\right.$, $P=0.99)$ were reliable. Interestingly, when correct and incorrect trials were combined, the overall CRT was significantly longer in the MUSC-dHPC condition compared with that of both MUSCmPFC and no infusion conditions (Fig. 6D). An ANOVA with repeated measures on two factors, with infusion condition (no infusion, MUSC-mPFC, and MUSC-dHPC) and delay (3-, 30-, and $60-\mathrm{sec}$ ) as repeated measures, revealed a significant main effect of condition $\left(F_{(2,28)}=70.79, P<0.001\right)$ and a significant infusion $\times$ delay interaction $\left(F_{(4,56)}=9.26, P<0.001\right)$, but not main effect of delay $\left(F_{(2,28)}=1.03, P=0.37\right)$. Tukey's post hoc comparisons demonstrated that rats exhibited a significantly longer overall CRT when muscimol was infused into the dHPC than when muscimol was infused into the mPFC or with no infusions $(P<0.001)$.

When another variable, the "back edge error," was analyzed (Fig. 7A), the MUSC-dHPC condition produced a significantly higher incidence of exploring the opposite edge runway (instead of directly returning to the center arm) than either the MUSCmPFC or ACSF infusion conditions (Fig. 7B). Data from two control conditions (ACSF-mPFC and ACSF-dHPC) were not different from each other and were thus pooled. An ANOVA with repeated measures on two factors, with infusion (ACSF, MUSC-mPFC, and MUSC-dHPC) and delay (3-, 30-, and 60-sec) as repeated measures, revealed significant main effects of condition $\left(F_{(2,22)}=27.04, P<0.001\right)$ and delay $\left(F_{(2,22)}=4.20, P=0.03\right)$ and a marginally significant infusion $X$ delay interaction $\left(F_{(4,44)}=2.50, P=0.056\right)$. Tukey's post hoc comparisons demonstrated that rats exhibited a significantly higher percentage of back edge errors with muscimol infusions into the dHPC compared with other infusion conditions $(P<0.001)$.

The final dependent variable analyzed was the percentage of incorrect arm choices made in consecutive trials (Fig. 8). For this, the percent proportions of two consecutive incorrect trials were compared between MUSC-mPFC and MUSC-dHPC conditions across delays. An ANOVA with repeated measures on two factors, with infusion condition (MUSC-mPFC and MUSC-dHPC) and delay (3-, 30-, and 60-sec) as repeated measures, revealed a significant main effect of infusion $\left(F_{(1,9)}=5.26, P=0.048\right)$, but failed to reveal a significant main effect of delay $\left(F_{(2,18)}=1.17, P=0.33\right)$ or a significant infusion $\times$ delay interaction $\left(F_{(2,18)}=0.55\right.$, $P=0.58)$. Unlike MUSC infusions that produced significant and comparable levels of incorrect trials, ACSF (control) infusions yielded marginal errors and thus were inappropriate for this analysis.

\section{Discussion}

The present study investigated the role of the PFC and the hippocampus in working memory while rats performed a spatial DAT on an automated figure-eight maze. Muscimol inactivation of either structure significantly reduced the working memory ac- 
A
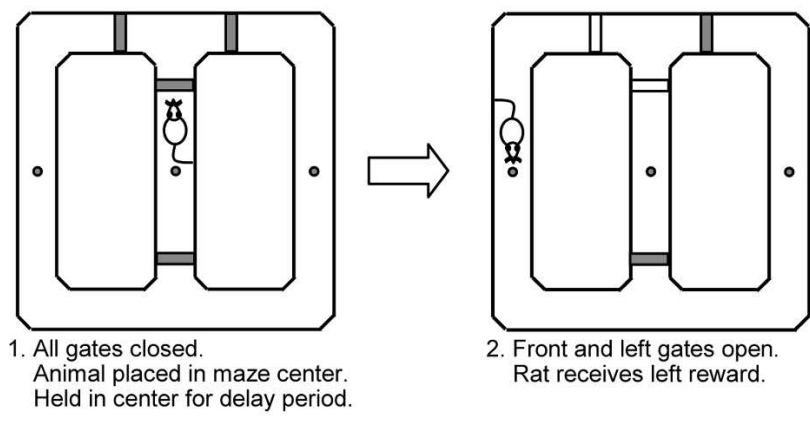

2. Front and left gates open. Rat receives left reward.
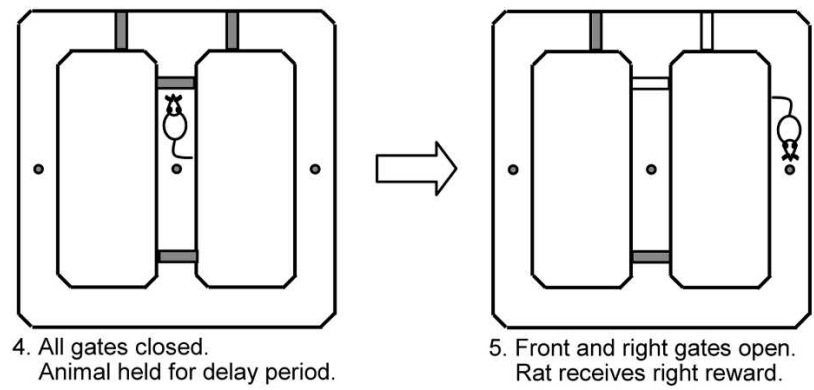

$\mathrm{B}$
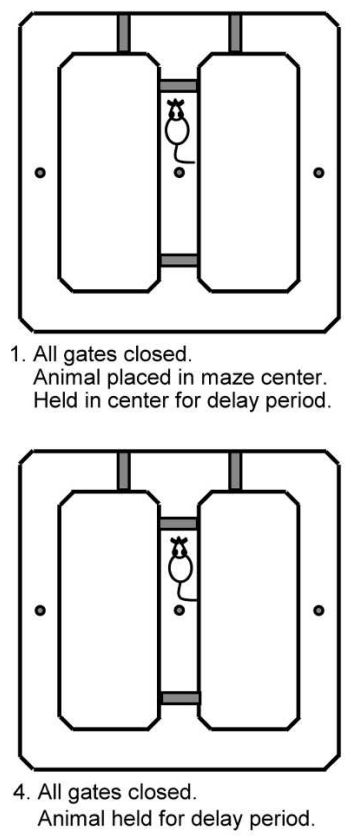
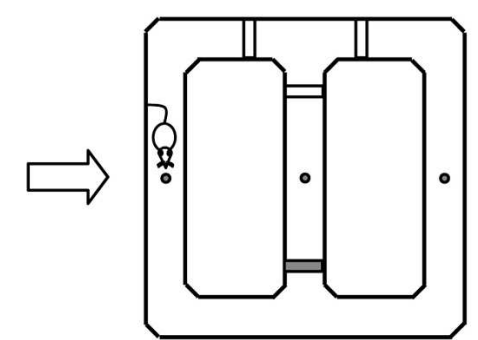

2. Front, left and right gates open. e.g., Rat CHOOSES left arm. Rewarded on choice alternation.
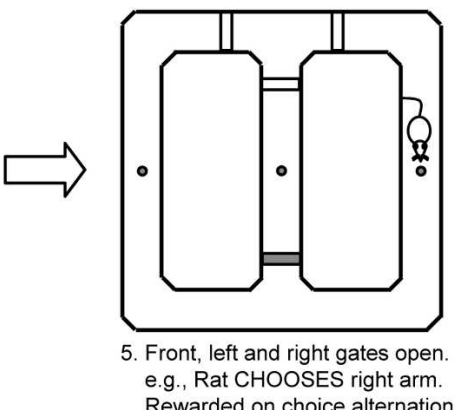
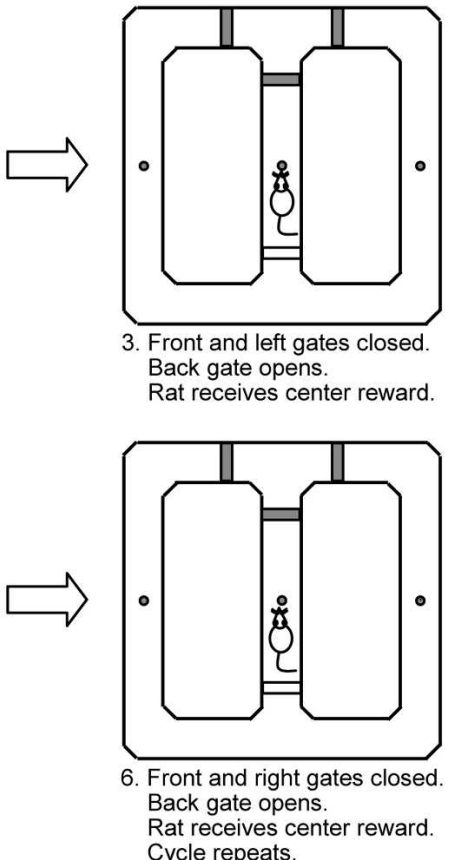

Cycle repeats.
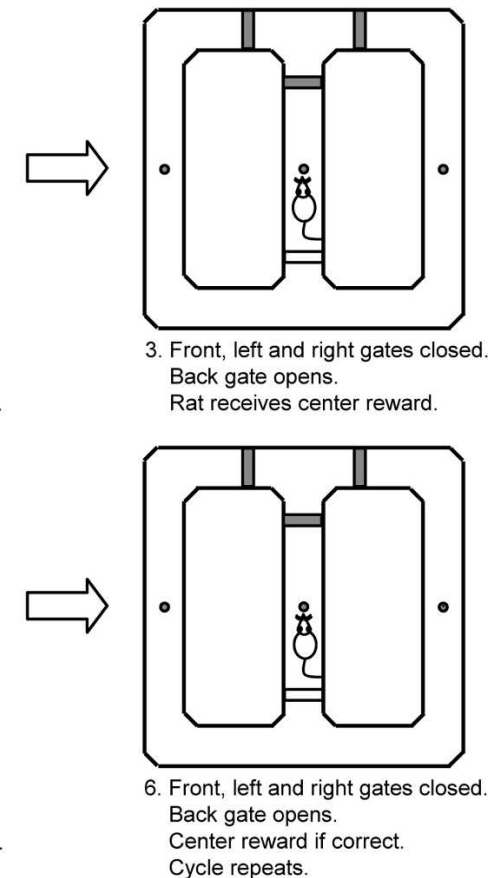

Figure 2. Illustration of a programmed DAT training procedure on the figure-eight maze. Sequence of gate operation was controlled by the computer based on the animal position on the maze during shaping $(A)$ and testing $(B)$.

curacy at all delay intervals, indicating that PFC or hippocampal functioning alone is insufficient to preserve performance on the DAT; instead, the interaction of the two structures is necessary for normal working memory. Our data further suggest that the PFC and hippocampus process qualitatively different information during the DAT.

By carefully inspecting performance on the DAT under PFC and hippocampus inactivation states, one can reasonably infer the default (intact brain) state of information processing required for working memory. Specifically, if the PFC and the hippocampus process information in parallel (and redundantly) or competitively, then either structure alone should be capable of supporting the DAT (see Kim and Baxter 2001). However, if two brain systems process different information but require interaction to produce coherent behavior required of the DAT, then both the PFC and hippocampus have to be functional. Since the inactivation of either the mPFC or dHPC severely impaired working memory, our data support the latter view; that is, the PFC and 
A

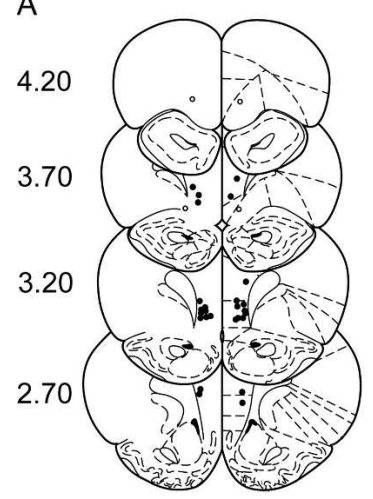

B

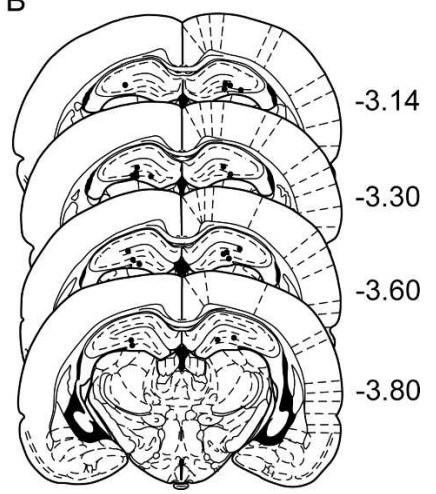

Figure 3. Histological reconstructions of cannula placement site. (Filled circles) Locations where infusion cannula tips were placed within the medial prefrontal cortex (mPFC: prelimbic and infralimbic) $(A)$ and the dorsal hippocampus (dHPC) (B). (Open circles) incorrectly placed cannula tip locations. Numbers indicate the distance in millimeters relative to bregma. Reprinted with permission from Elsevier (01997, Paxinos and Watson 1997.

the hippocampus interact in a synergistic manner during the DAT. A recent study also found that functional disconnection between the hippocampus and the PFC impaired spatial working memory in rats (Wang and Cai 2006).

The PFC and hippocampus may interact via their direct/ indirect anatomical connections (Ferino et al. 1987; Laroche et al. 2000; Thierry et al. 2000). Consistent with anatomy, electrophysiological studies have shown that cellular activities in these two regions are intimately coordinated during working memory tasks (Hyman et al. 2005; Siapas et al. 2005). Specifically, hippocampus and PFC activities are closely correlated in time by virtue of hippocampal theta rhythms. Therefore, theta rhythms might orchestrate spatial memory and working memory that are managed mainly by the hippocampus and PFC, respectively. Recently, Jones and Wilson (2005) found that the correlation of cell firings in the PFC and the hippocampus improves as the demand for spatial working memory increases. Overall, these cellular characteristics are consistent with behavioral results of the present study.

Our data show that the inactivation of the hippocampus disrupts the DAT at 3-, 30-, and 60-sec delays, which is consistent with earlier studies indicating the importance of the hippocampus in working memory functioning at short as well as long delays (Kesner et al. 1996; Hampson et al. 1999). In a related study, Lee and Kesner (2003) tested spatial working memory in rats using a radial eight-arm maze, but they found that hippocampal function in spatial working memory becomes critical only when the delay is relatively long (see also Mizumori et al. 1987 with the protein synthesis inhibitor anisomycin). A key procedural difference in their study is that after surgery, training sessions were reinstated before testing started; thus, additional training sessions may have activated an alternate system for the task. Interestingly, they also reported a significant performance deficit with hippocampal lesions during the initial training sessions after surgery, which is consistent with our findings.

At present, the notion of delay-dependent involvement of the PFC in working memory remains equivocal. While some studies show delay-independent impairment of working memory with the disruption of the PFC (Chudasama and Muir 1997; Porter et al. 2000), others show delay-dependent deterioration of working memory (Delatour and Gisquet-Verrier 1996; Sloan et al. 2006). Contrasting results may be attributable to different lesion methods (electrolytic vs. excitotoxic vs. radiofrequency vs. aspi-

ration) and behavioral tasks (lever pressing vs. radial arm maze). It is also worth considering that the role of the PFC in working memory may depend on the structure of behavioral task. That is, the working memory necessary to complete a continuous foraging task (such as an eight-arm radial maze) can be supported by other brain areas. However, the PFC is necessary when the task is divided into two halves and a long delay is imposed between them (Bubser and Schmidt 1990; Floresco et al. 1997). An interesting finding by Porter et al. (2000) shows that mPFC lesions produce a delay-dependent working memory deficit in the alternating arm choice response (the same two arms available repeatedly) on a radial arm maze when extramaze spatial cues are available. However, the deficit becomes delay-independent when spatial information is not provided. A differential working memory deficit based on the availability of extramaze spatial cues suggests that animals with the disrupted PFC are capable of completing the task at a short delay using an alternate strategy (i.e., spatial/ episodic memory) provided by the intact hippocampus, but exhibit impaired performance at longer delays as memory demands increase.

An interesting novel finding from our study is that the difference in CRT for correct vs. incorrect trials (observed prior to drug manipulation) disappeared with muscimol infusions into the mPFC and dHPC. The level of CRT, measured by the time taken from the start of each trial to the completion of arm choice response, may reflect the "confidence" level of the animal in making its choice response for a reward. Specifically, the inactivation of the mPFC or dHPC produced equivalent CRT for correct and incorrect trials. The lack of difference in CRT between correct and incorrect trials with muscimol suggests that the observed "correct" choice responses in fact may be due to "chance" performance rather than a product of intact memory functioning. If true, future ensemble unit recording studies should be able to discern the intact memory-derived "true correct" choice responses from the coincidental performance-derived "false correct" choice responses.

Another notable finding is that when muscimol was infused into the dHPC (but not the mPFC), animals made significantly more frequent back edge errors (i.e., exploring the opposite edge runway instead of entering the center holding area). The back edge error provides valuable information concerning the roles of the mPFC and dHPC in spatial reference memory (SRM) for the task. During shaping and testing procedures in our figure-eight maze, the animal's SRM may include departing the center holding area when the front gate drops, making an arm choice response to receive water reward (at specific locations on the maze), and returning to the center area for the next trial. The inactivation of the AHPC also drastically increased the overall CRT; rats seemed spatially disoriented during the delay period
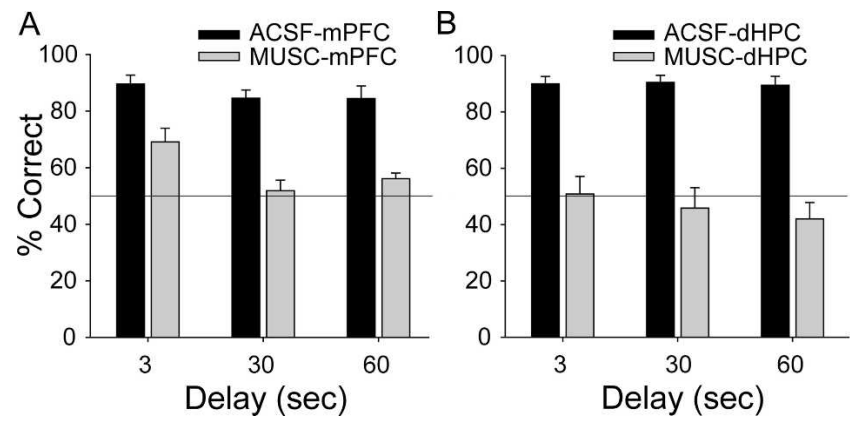

Figure 4. Effects of muscimol infusions on DAT performance. Mean $( \pm \mathrm{SE})$ percentage of correct trials at 3-, 30-, and 60-sec delay periods when ACSF (black) and MUSC (gray) were microinfused into the mPFC $(A)$ and the dHPC (B). (Horizontal line) Chance level of performance. 
A
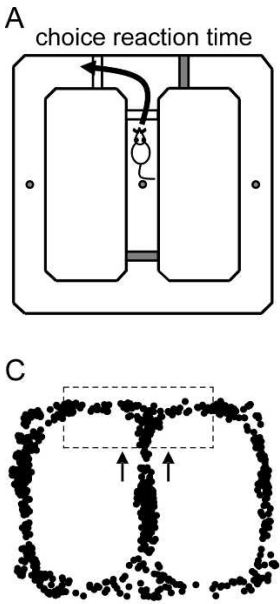

First day
B

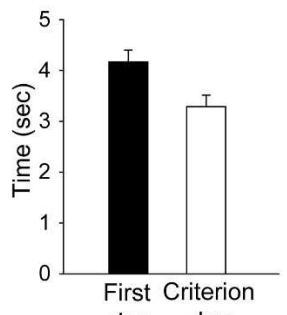

day day

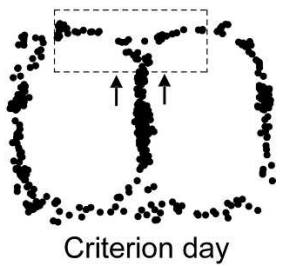

Criterion day

Figure 5. Arm choice reaction time (CRT) as a function of training. $(A)$ CRT was quantified as the latency from the start of a trial (front and side gates down) to when the animal made an arm choice response (side gates up). (B) Mean ( \pm SE) CRT during the first testing session (black) and the criterion testing session (white). (C) Sample movement trajectory maps show decrease in choice response variability (dash box) during the first testing day vs. criterion day.

and thus required a longer time to initiate the arm choice response (Fig. 6D). These data are consistent with previous studies that found disruption of SRM following hippocampal lesions when tested in water and radial arm maze tasks (Cassel et al. 1998; Pothuizen et al. 2004; Sloan et al. 2006), and intact SRM after mPFC lesions (Granon et al. 1994; Sloan et al. 2006). In most studies, hippocampal lesions produce not only SRM but also spatial working memory impairments, especially when the memory demand for allocentric spatial information is strong (for a recent review, see Dudchenko 2004). The mPFC then must retrieve allocentric spatial information from the hippocampus as needed. Consistent with this possibility, single-unit recording studies found no evidence for allocentric spatial information in the mPFC (Poucet 1997; Jung et al. 1998). Accordingly, the hippocampus may serve the role of episodic buffer (i.e., integrate present and preceding information to properly anticipate future experience), a newly added component of working memory (Baddeley 2000, 2003).

Hippocampal inactivation also increased the perseveration of choice response, revealed by a rise in the percentage of two consecutive incorrect trials (Fig. 8). In contrast, with mPFC inactivation, although animals performed at a near chance level, there were a higher percentage of correct trials than incorrect trials across delays following incorrect trials, suggesting that animals adjusted their choice response when an incorrect choice was made on the previous trial. These findings are consistent with earlier studies demonstrating response perseveration with damage to the hippocampus (Devenport et al. 1981; Mickley et al. 1989; Whishaw and Tomie 1997), but not with damage to the PFC (Granon et al. 1994; Seamans et al. 1995; Delatour and Gisquet-Verrier 2000). Interestingly, although PFC lesions did not produce response perseveration for a choice alternation response when animals were tested during a single session, the perseveration occurred when animals were required to shift the response rule (e.g., reversal learning) between sessions (Seamans et al. 1995; Dias and Aggleton 2000).

It should be noted that although hippocampal inactivation produced perseveration of choice responses in the DAT, it is not clear from the present study whether the increased perseveration is related to the disruption of working memory system per se or

is an altered behavioral pattern produced independently of muscimol effect on working memory. According to the relational learning theory (Squire 1987; Cohen and Eichenbaum 1993), the hippocampus plays a critical role in learning relationships between stimuli or events. Therefore, animals can use a series of relations to create an episodic memory. When the hippocampus is damaged, the relational memory provided by the hippocampus is disrupted, and animals may perform the task using only a portion of information detached from the whole episode. Thus, the increased perseverative behavior observed here may be attributed to the disruption of the hippocampal role in relational memory.

However, it is also possible that hippocampal (but not PFC) inactivation might have impaired the neural system for the natural tendency of alternation responses. Generally, animals tend to explore alternate routes rather than taking a previously tested path to reach a goal (Douglas and Isaacson 1964; O'Keefe and Nadel 1978). This natural behavioral pattern (of "win-shift") might have been disrupted by hippocampal inactivation as shown in the present study. Although it remains unclear how the hippocampal function is involved in the selection of choice responses, the nucleus accumbens, which is anatomically connected to the hippocampus, might provide necessary information about the behavioral pattern to the hippocampus. For example, it has been shown that the nucleus accumbens is critically involved in a motor strategy for goal-directed learning tasks (Morgenson et al. 1993; Seamans and Phillips 1994). Therefore, hippocampal inactivation can potentially disrupt information needed by the nucleus accumbens to appropriately adjust the goal-directed behavior.

A couple of caveats should be mentioned about the present study. First, although the DAT (using T and figure-eight mazes) is a widely accepted test of working memory in rodents (see Pedigo et al. 2006), we have not tested whether a distractive cue during
A
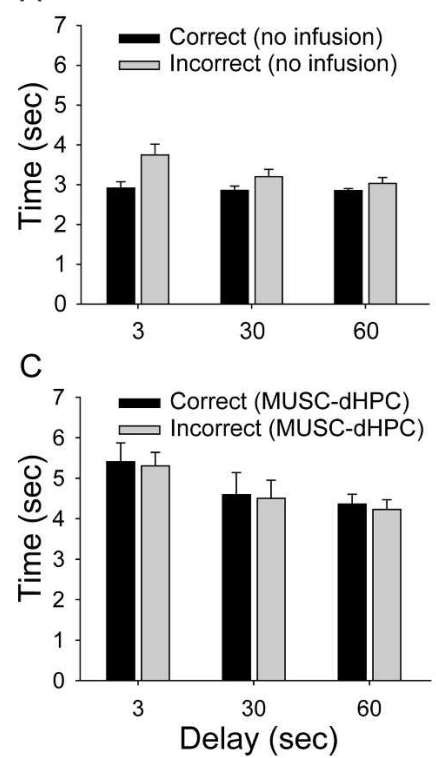

B

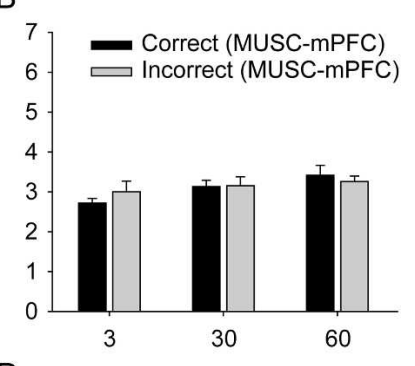

$\mathrm{D}$

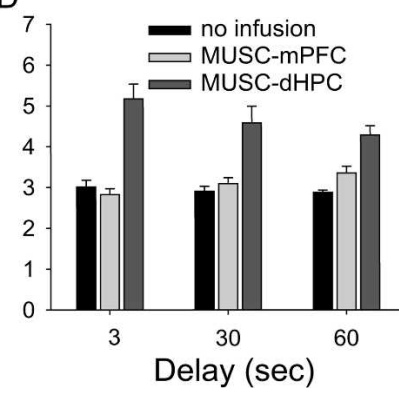

Figure 6. Effects of muscimol infusions on CRT. $(A)$ Mean $( \pm S E)$ CRT for correct (black) and incorrect (gray) trials during testing sessions with no drug infusion (Day 1$)$ at various delays. ( $B)$ Mean ( \pm SE) CRT for correct (black) and incorrect (gray) trials in MUSC-mPFC animals. (C) Mean ( \pm SE) CRT for correct (black) and incorrect (gray) trials in MUSCdHPC animals. (D) Mean ( \pm SE) CRT (correct and incorrect trials combined) with no drug infusion (black), MUSC-mPFC (light gray), and MUSC-dHPC (dark gray) conditions at various delays. 
A

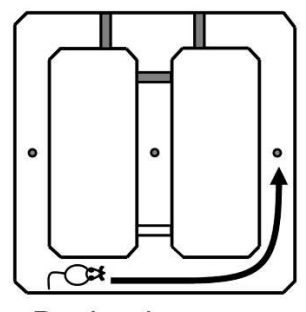

Back edge error
B

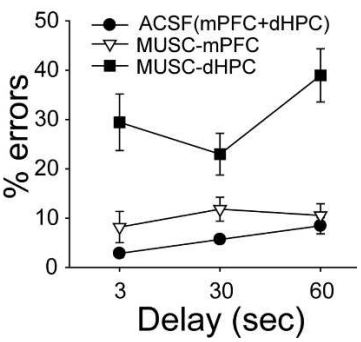

Figure 7. Effects of muscimol infusions on "back edge errors." $(A)$ Back edge error was made when the animal entered the opposite edge runway rather than returning to the center holding area. $(B)$ Mean $( \pm S E)$ percentage of back edge errors made by ACSF(mPFC+dHPC), MUSC-mPFC, and MUSC-dHPC conditions.

a delay period interferes with the performance on the figureeight maze. Given that a distractive stimulus during a delay period has been shown to disrupt spatial as well as nonspatial working memory in rats (e.g., Ennaceur and Meliani 1992), if the DAT is not affected by distractive cues, then this would suggest that other memory systems (perhaps long-term memory or motor memory) are also engaged in the present task. Second, the observed differences in behavior measures between mPFC and dHPC inactivation can be due to possible differences in muscimol spread or the tissue responsivity to the drug concentration used. Although mPFC lesions also produced selective deficits in working (but not reference) memory on the same maze (Pedigo et al. 2006), this latter possibility cannot entirely be excluded.

In summary, the present findings indicate that either the PFC or the hippocampus alone is not sufficient to support spatial working memory in rats. Instead, a functional interaction between the two structures is necessary. It is likely that the hippocampus provides the critical spatial/episodic information to the PFC that is necessary for the successful execution of the DAT (Fig. 9). However, hippocampal function alone is not sufficient for the DAT since mPFC inactivation leads to selective disruption of working memory processing. Our data further show that the inactivations of the mPFC and dHPC influence different aspects of DAT performance. The distinct behavioral effects include CRT (in making the arm choice response), back edge error (reflecting reference memory), and response perseveration (perhaps a shift in strategy), all of which were significantly altered by inactivating the dHPC (but not the PFC). Although dHPC inactivation produced wide-ranging behavioral alterations (previously unreported) rather than working memory per se, the fact that the animals still demonstrated some ability to perform the task (despite the errors) indicates the cognitive complexities and the dynamic engagement of multiple memory systems in the DAT.

\section{Materials and Methods}

\section{Subjects}

Fifteen experimentally naïve male Sprague Dawley rats (initially weighing 250-275 g) were individually housed in our Association for Assessment and Accreditation of Laboratory Animal Care (AAALAC) accredited animal care facility and maintained on a reverse 12-h light/dark cycle (lights on at 7:00 p.m.). A week prior to and for the duration of the experiment, daily water access was restricted to maintain $85 \%$ of the animal's normal body weight. The food pellet was available ad libitum in the home cage. All experiments were conducted during the dark phase of the cycle and in strict compliance with the University of Washington Institutional Animal Care and Use Committee guidelines.

\section{Surgery}

Animals were anesthetized via intraperitoneal injection of a 30 $\mathrm{mg} / \mathrm{kg}$ ketamine and $2.5 \mathrm{mg} / \mathrm{kg}$ xylazine mixture, with supplemental injections given as needed. Under aseptic conditions, a stereotaxic instrument with nonpuncture ear bars (Stoelting) was used to implant 26-gauge guide cannulae (Plastic One) bilaterally into the mPFC (from bregma, $3.20 \mathrm{~mm}$ anterior, $\pm 2.21 \mathrm{~mm}$ lateral, $3.26 \mathrm{~mm}$ ventral; the cannulae were angled $20^{\circ}$ from a vertical midline) and dHPC (from bregma, $3.60 \mathrm{~mm}$ posterior, $\pm 3.24 \mathrm{~mm}$ lateral, $2.62 \mathrm{~mm}$ ventral). Implanted cannulae were cemented to six anchoring screws on the skull. Dummy cannulae were inserted into the implanted cannulae (protruding $0.5 \mathrm{~mm}$ ) to maintain the patency of the guide cannulae. During 7-10 d of postoperative recovery, animals were adapted to handling, and each dummy cannula was removed and replaced with a clean one.

\section{Drugs and infusion}

Muscimol free base (Sigma-Aldrich), dissolved in artificial cerebrospinal fluid (ACSF) $(10 \mathrm{mM}, \mathrm{pH} \sim 7.4)$ was microinfused bilaterally into either the mPFC or dHPC by back-loading the drug up a 33-gauge infusion cannula into polyethylene (PE20) tubing connected to $10-\mu \mathrm{L}$ Hamilton microsyringes (Hamilton Company). The infusion cannula protruded $1 \mathrm{~mm}$ beyond the guide cannula. An infusion volume of $0.3 \mu \mathrm{L}$ (per side) was delivered using a Harvard 11 Plus Syringe Pump (Harvard Apparatus) over the course of $3 \mathrm{~min}$ (at a rate of $0.1 \mu \mathrm{L} / \mathrm{min}$ ). The infusion cannula remained in place for an additional $1 \mathrm{~min}$ after the infusions before being pulled out.

The muscimol dosage $(0.3 \mu \mathrm{g} / 0.3 \mu \mathrm{L}$ per side $)$ was based on previous studies from our laboratory (Kim et al. 2005) and others (Wilensky et al. 1999; Maren et al. 2001; Lee and Kesner 2003). Based on studies that examined ${ }^{3} \mathrm{H}$-muscimol spreading (Krupa et al. 1996; Arikan et al. 2002) in the cerebellum in which a 1- $\mu \mathrm{L}$ volume infusion diffused a radius of $1.6-2.0 \mathrm{~mm}$, it was estimated that $0.3 \mu \mathrm{L}$ of muscimol used in the present study would spread within a radius of $\sim 0.5-0.7 \mathrm{~mm}$ from the infusion needle tip. Hence, it is likely that infused muscimol would have diffused to most of infralimbic and prelimbic areas of the prefrontal cortex, and to most of the dHPC with respective injections and possibly to minor portions of adjacent neighboring structures.

\section{Maze apparatus and data collection}

The dimensions and automatic features of the figure-eight maze have previously been described in detail (Pedigo et al. 2006). In brief, the maze (elevated $0.75 \mathrm{~m}$ from the floor) is in the shape of an open square with a center bridge runway that connects the front edge runway with the back edge runway (Fig. 1). The maze has four acrylic gates, actuated by air-regulated pneumatic cylinders, that ascend (30 $\mathrm{cm}$ above the maze surface) and descend (leveled to the maze surface) to block or allow the animal to pass. Two gates at either end of the center bridge imposed delays be-

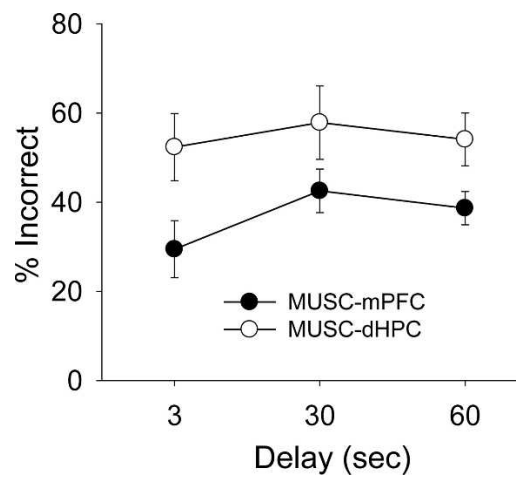

Figure 8. Effects of muscimol infusions on two consecutive incorrect choices. Mean $( \pm \mathrm{SE})$ percent of incorrect trials immediately following incorrect trials across delays when muscimol was infused into the MPFC (black) and dHPC (white). 


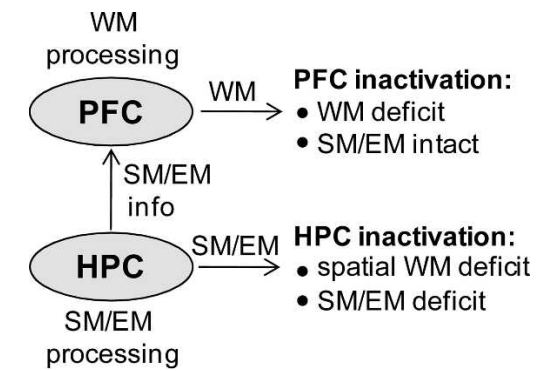

Figure 9. A hypothetical model of neural structures involved in working memory (WM). The model posits that the hippocampus (HPC) is involved in processing spatial memory (SM) and episodic memory (EM), which are used by the PFC in spatial working memory tasks. Accordingly, inactivation of the HPC will lead to WM, SM, and EM deficits, while inactivation of the PFC will produce relatively specific WM impairments.

tween trials. The water reward $(0.04 \mathrm{~mL})$ was delivered (at three small orifices shown) through a stainless steel liquid tubing assembly connected, via $24-\mathrm{V}$ electric solenoid valves, to a water storage vessel. A digital input/output card (PCI-6503; National Instruments) was used to control the solenoid valves and pneumatic cylinders, and an analog video frame grabber card (PCI1405) was used to capture images from a camera over the maze. The maze system was controlled by an Intel Pentium IV processor $\mathrm{PC}$, using custom software written in the "C" programming language; the compiler for the " $\mathrm{C}$ " code is National Instruments Lab Windows CVI (version 5.0). The software calculated and created a time-stamped data file with the following dependent variables: the animal location and activity, gate status, elapsed time, choice accuracy, and distance traveled over time. A video clip of a rat performing the DAT is available for viewing at http://faculty. washington.edu/jeansokk/research\%20new.htm.

\section{Behavioral procedures}

Animals underwent the following three successive stages of maze training before the final testing phase (cf. Pedigo et al. 2006).

\section{Habituation}

For the first $3 \mathrm{~d}$, animals were brought to the maze area in transport boxes and remained there for $2 \mathrm{~h}$ each day. The maze was programmed to actuate the gates in a pseudorandom sequence to help the animals get habituated to the noise of the maze operation, background white noise, and light settings. On the fourth day, animals were placed in the center holding area of the maze and confined by the front and back gates. During a single 30-min session, both side gates were operational in a pseudorandom sequence.

\section{Shaping}

Animals were placed in the center holding area of the maze with all four gates in the "UP" position (Fig. 2A). After a 3-sec delay, the front and one of the side gates were dropped, allowing the animal to leave the center area and turn to one side (e.g., left) of the maze. At the instant the animal passed the left gate, the front and left gates were raised, and the back gate (on the center runway) was dropped. Upon reaching the left water port location, the animal was rewarded. The animal was allowed to freely explore the rest of the maze (with the two side gates and the front gate in the "UP" position). When the animal entered the center holding area, it was rewarded with water at the center port and the back gate was elevated. The delay period started, and the same sequence of water and gate operation was repeated with the alternate side gate (from the previous trial) in the "DOWN" position. During shaping, the animal had only one choice to make; as it approached the center area along the back edge, it could either enter the center or continue on to the opposite side of the maze (in which case access to the center area via the front edge was blocked). The entering of the opposite edge runway was defined as a "back edge error," which was used as an index to gauge how well the animal learned the general maze procedure and to determine when shaping was completed. For the present study, animals were shaped once a day with a 3-sec center delay until they reached 40 laps in $<30$ min with four or fewer back edge errors ( $\leq 10 \%)$. Upon meeting this criterion (usually $2-3 \mathrm{~d}$ ), animals advanced to the testing phase.

\section{Testing}

The animals were trained to make alternate arm choice responses to obtain the water reward (Fig. 2B). After a 3-sec delay in the center holding area, the front, left, and right gates dropped. The animal then had the freedom to make either a left or a right turn. Once the animal made a turn, the front, left, and right gates were elevated (and the back gate was dropped). The animal was rewarded at the water port location (e.g., left). When the animal returned to the center area, it was rewarded with water at the center water port, and the back gate was elevated. The delay period started again once the back gate was fully elevated, and the sequence was repeated for additional laps. In subsequent laps, correct arm choice (e.g., left $\rightarrow$ right) resulted in reward, whereas incorrect arm choice (e.g., left $\rightarrow$ left) resulted in the absence of reward (at both side and center water ports). The testing session ended when 40 trials were completed or $30 \mathrm{~min}$ elapsed, whichever came first. Once the animal reached the criterion of 30 or more correct trials $(\geq 75 \%)$, the final testing phase started the following day.

\section{Testing with drug infusions}

On Day 1 of the final phase of testing, animals received two 20 trial sessions that were separated by $90 \mathrm{~min}$, which is the same interval as drug infusion sessions. The Day 1 sessions were to ensure that (1) rats could perform comparably during the two sessions and (2) rats could perform well with increased delays. Performance during these sessions served as a baseline measure to compare sessions with drug infusions. On Day 2, ACSF $(0.3 \mu \mathrm{L})$ was infused bilaterally into the mPFC (or dHPC), and $30 \mathrm{~min}$ later animals underwent a 20 -trial testing session. Ninety minutes later, muscimol was infused bilaterally into the mPFC (or dHPC), and then animals underwent another 20-trial testing session. On the following day, the recovery of performance from drug infusions was tested in a single 20-trial session before advancing to the next infusion site. After both infusion sites had been tested, the delay period in the center holding area was increased from $3 \mathrm{sec}$ to $30 \mathrm{sec}$, and then to $60 \mathrm{sec}$.

\section{Histology}

At the completion of behavioral testing, animals were overdosed with urethane and perfused intracardially with $0.9 \%$ saline followed by $10 \%$ buffered formalin. The brains were removed and stored in $10 \%$ formalin overnight and then kept in 30\% sucrose solution until they sank. Transverse sections $(50 \mu \mathrm{m})$ were taken through the extent of the cannula tract, mounted on gelatincoated slides, and stained with cresyl violet to verify cannula placements.

\section{Data analysis}

Statistical comparisons between groups were examined using Student's $t$-tests (paired) or ANOVA (cf. Winer et al. 1991). For a significant difference $(P<0.05)$, post hoc comparisons were performed using Tukey's honestly significant difference (HSD) tests. For choice reaction time (CRT) analyses, values that were outside the range of \pm 3 SDs around the group mean were regarded as outliers and excluded from the analyses.

\section{Acknowledgments}

We thank Samuel Pedigo for his valuable assistance in the automation of the maze. This work was supported by NIMH (MH64457) and the University of Washington Royalty Research Fellowship to J.J.K. 


\section{References}

Amaral, D.G. and Witter, M.P. 1995. Hippocampal formation. In The rat nervous system (ed. G. Paxinos), pp. 443-493. Academic Press, San Diego, CA.

Arikan, R., Blake, N.M., Erinjeri, J.P., Woolsey, T.A., Giraud, L., and Highstein, S.M. 2002. A method to measure the effective spread of focally injected muscimol into the central nervous system with electrophysiology and light microscopy. J. Neurosci. Methods 118: $51-57$.

Baddeley, A.D. 1986. Working memory. Clarendon, Oxford.

Baddeley, A. 2000. The episodic buffer: A new component of working memory? Trends Cogn. Sci. 4: 417-423.

Baddeley, A. 2003. Working memory: Looking back and looking forward. Nat. Rev. Neurosci. 4: 829-839.

Baddeley, A.D. and Hitch, G.J. 1974. Working memory. In Recent advances in learning and motivation (ed. G.A. Bower), Vol. 8, pp. 47-90. Academic Press, New York.

Baeg, E.H., Kim, Y.B., Huh, K., Mook-Jung, I., Kim, H.T., and Jung, M.W. 2003. Dynamics of population code for working memory in the prefrontal cortex. Neuron 40: 177-188.

Baeg, E.H., Kim, Y.B., Kim, J., Ghim, J.W., Kim, J.J., and Jung, M.W 2007. Learning-induced enduring changes in functional connectivity among prefrontal cortical neurons. J. Neurosci. 27: 909-918.

Birnbaum, S.G., Yuan, P.X., Wang, M., Vijayraghavan, S., Bloom, A.K., Davis, D.J., Gobeske, K.T., Sweatt, J.D., Manji, H.K., and Arnsten, A.F. 2004. Protein kinase C overactivity impairs prefrontal cortical regulation of working memory. Science 306: 882-884.

Bubser, M. and Schmidt, W.J. 1990. 6-Hydroxydopamine lesion of the rat prefrontal cortex increases locomotor activity, impairs acquisition of delayed alternation tasks, but does not affect uninterrupted tasks in the radial maze. Behav. Brain Res. 37: 157-168.

Cassel, J.C., Cassel, S., Galani, R., Kelche, C., Will, B., and Jarrard, L. 1998. Fimbria-fornix vs selective hippocampal lesions in rats: Effects on locomotor activity and spatial learning and memory. Neurobiol. Learn. Mem. 69: 22-45.

Chudasama, Y. and Muir, J.L. 1997. A behavioral analysis of the delayed non-matching to position task: The effects of scopolamine, lesions of the fornix and of the prelimbic region on mediating behaviors by rats. Psychopharmacology 134: 73-82.

Clinton, S.M., Sucharski, I.L., and Finlay, J.M. 2006. Sesipramine attenuates working memory impairments induced by partial loss of catecholamines in the rat medial prefrontal cortex. Psychopharmacology 183: 404-412.

Cohen, N.J. and Eichenbaum, H. 1993. Memory, amnesia, and the hippocampal system. The MIT Press, Cambridge, MA.

Delatour, B. and Gisquet-Verrier, P. 1996. Prelimbic cortex specific lesions disrupt delayed-variable response tasks in the rat. Behav. Neurosci. 110: 1282-1298.

Delatour, B. and Gisquet-Verrier, P. 2000. Functional role of rat prelimbic-infralimbic cortices in spatial memory: Evidence for their involvement in attention and behavioural flexibility. Behav. Brain Res. 109: 113-128.

Devenport, L.D., Devenport, J.A., and Halloway, F.A. 1981. Reward-induced stereotypy: Modulation by the hippocampus. Science 212: $1288-1289$.

Dias, R. and Aggleton, J.P. 2000. Effects of selective excitotoxic prefrontal lesions on acquisition of nonmatching- and matching-to-place in the T-maze in the rat: Differential involvement of the prelimbic-infralimbic and anterior cingulate cortices in providing behavioural flexibility. Eur. J. Neurosci. 12: 4457-4466.

Douglas, R.J. and Isaacson, R.L. 1964. Hippocampal lesions and activity. Psychon. Sci. 1: 187-188.

Dudchenko, P.A. 2004. An overview of the tasks used to test working memory in rodents. Neurosci. Biobehav. Rev. 28: 699-709.

Eichenbaum, H. 2000. A cortical-hippocampal system for declarative memory. Nat. Rev. Neurosci. 1: 41-50.

Ennaceur, A. and Meliani, K. 1992. A new one-trial test for neurobiological studies of memory in rats. III. Spatial vs. non-spatial working memory. Behav. Brain Res. 51: 83-92.

Ferino, F., Thierry, A.M., and Glowinski, J. 1987. Anatomical and electrophysiological evidence for a direct projection from Ammon's horn to the medial prefrontal cortex in the rat. Exp. Brain Res. 65: $421-426$

Floresco, S.B., Seamans, J.K., and Phillips, A.G. 1997. Selective roles for hippocampal, prefrontal cortical, and ventral striatal circuits in radial-arm maze tasks with or without a delay. J. Neurosci. 17: $1880-1890$.

Funahashi, S., Bruce, C.J., and Goldman-Rakic, P.S. 1989. Mnemonic coding of visual space in the monkey's dorsolateral prefrontal cortex. J. Neurophysiol. 61: 331-349.

Fuster, J.M. 1997. Network memory. Trends Neurosci. 20: 451-459.
Fuster, J.M. 2001. The prefrontal cortex-An update: Time is of the essence. Neuron 30: 319-333.

Fuster, J.M. and Alexander, G.E. 1971. Neuron activity related to short-term memory. Science 173: 652-654.

Goldman-Rakic, P.S. 1987. Circuitry of primate prefrontal cortex and regulation of behavior by representational memory. In Handbook of physiology (ed. F. Plum), Vol. 5, pp. 373-417. American Physiological Society, Bethesda, MD.

Goldman-Rakic, P.S. 1996. Regional and cellular f, MDractionation of working memory. Proc. Natl. Acad. Sci. 93: 13473-13480.

Granon, S., Vidal, C., Thinus-Blanc, C., Changeux, J.P., and Poucet, B. 1994. Working memory, response selection, and effortful processing in rats with medial prefrontal lesions. Behav. Neurosci. 108: 883-891.

Hampson, R.E., Jarrard, L.E., and Deadwyler, S.A. 1999. Effects of ibotenate hippocampal and extrahippocampal destruction on delayed-match and -nonmatch-to-sample behavior in rats. $J$. Neurosci. 19: 1492-1507.

Hyman, J.M., Zilli, E.A., Paley, A.M., and Hasselmo, M.E. 2005. Medial prefrontal cortex cells show dynamic modulation with the hippocampal theta rhythm dependent on behavior. Hippocampus 15: 739-749.

Jarrard, L.E. 1993. On the role of the hippocampus in learning and memory in the rat. Behav. Neural Biol. 60: 9-26.

Jones, M.W. and Wilson, M.A. 2005. Theta rhythms coordinate hippocampal-prefrontal interactions in a spatial memory task. PLoS Biol. 3: e402. doi: 10.1371/journal.pbio.0030402.

Jung, M.W., Qin, Y., McNaughton, B.L., and Barnes, C.A. 1998. Firing characteristics of deep layer neurons in prefrontal cortex in rats performing spatial working memory tasks. Cereb. Cortex 8: 437-450.

Kesner, R.P., Hunt, M.E., Williams, J.M., and Long, J.M. 1996. Prefrontal cortex and working memory for spatial response, spatial location, and visual object information in the rat. Cereb. Cortex 6: 311-318.

Kim, J.J. and Baxter, M.G. 2001. Multiple brain-memory systems: The whole does not equal the sum of its parts. Trends Neurosci. 24: 324-330.

Kim, J.J., Koo, J.W., Lee, H.J., and Han, J.S. 2005. Amygdalar inactivation blocks stress-induced impairments in hippocampa long-term potentiation and spatial memory. J. Neurosci. 25: $1532-1539$.

Kolb, B. 1990. Animal models for human PFC-related disorders. Prog. Brain Res. 85: 501-519.

Krupa, D.J., Thompson, J.K., and Thompson, R.F. 1996. Localization of a memory trace in the mammalian brain. Science 260: 989-991.

Kubota, K. and Niki, H. 1971. Prefrontal cortical unit activity and delayed alternation performance in monkeys. J. Neurophysiol. 34: 337-347.

Laroche, S., Davis, S., and Jay, T.M. 2000. Plasticity at hippocampal to prefrontal cortex synapses: Dual roles in working memory and consolidation. Hippocampus 10: $438-446$.

Lee, I. and Kesner, R.P. 2003. Time-dependent relationship between the dorsal hippocampus and the prefrontal cortex in spatial memory. $J$. Neurosci. 23: 1517-1523.

Maren, S., Yap, S.A., and Goosens, K.A. 2001. The amygdala is essential for the development of neuronal plasticity in the medial geniculate nucleus during auditory fear conditioning in rats. J. Neurosci. 21: RC135 (1-6).

Maruki, K., Izaki, Y., Hori, K., Nomura, M., and Yamauchi, T. 2001. Effects of rat ventral and dorsal hippocampus temporal inactivation on delayed alternation task. Brain Res. 895: 273-276.

Mickley, G.A., Ferguson, J.L., Nemeth, T.J., Mulvihill, M.A., and Alderks, C.E. 1989. Spontaneous perseverative turning in rats with radiation-induced hippocampal damage. Behav. Neurosci. 103: $722-730$.

Miller, E.K., Li, L., and Desimone, R. 1991. A neural mechanism for working and recognition memory in inferior temporal cortex. Science 254: $1377-1379$.

Mizumori, S.J., Channon, V., Rosenzwig, M.R., and Bennett, E.L. 1987. Anisomycin impairs long-term working memory in a delayed alternation task. Behav. Neural Biol. 47: 1-6.

Morgenson, G.J., Brudzynski, S.M., Wu, M., Yang, C.R., and Yim, C.Y. 1993. From motivation to action: A review of dopaminergic regulation of limbic $\rightarrow$ nucleus accumbens $\rightarrow$ ventral pallidum $\rightarrow$ pedunculopontine nucleus circuitries involved with limbic-motor integration. In Limbic-motor circuits and neuropsychiatry (eds. P.W. Kalivas and C.D. Barnes), pp 193-263. CRC Press, Boca Raton, FL.

Moser, E., Moser, M.B., and Andersen, P. 1993. Spatial learning impairment parallels the magnitude of dorsal hippocampal lesions, but is hardly present following ventral lesions. J. Neurosci. 13: $3916-3925$.

Murphy, B.L., Arnsten, A.F., Goldman-Rakic, P.S., and Roth, R.H. 1996. Increased dopamine turnover in the prefrontal cortex impairs spatia 
working memory performance in rats and monkeys. Proc. Natl. Acad. Sci. 93: $1325-1329$.

O'Keefe, J. and Nadel, L. 1978. The hippocampus as a cognitive map. Clarendon Press, Oxford.

Olton, D.S., Becker, J.T., and Handelmann, G.E. 1979. Hippocampus, space, and memory. Behav. Brain Sci. 2: 313-365.

Paxinos, G. and Watson, C. 1997. The rat brain in stereotaxic coordinates. Academic Press, San Diego, CA.

Pedigo, S.F., Song, E.Y., Jung, M.W., and Kim, J.J. 2006. A computer vision-based automated figure-8 maze for working memory test in rodents. J. Neurosci. Methods 156: $10-16$.

Porter, M.C., Burk, J.A., and Mair, R.G. 2000. A comparison of the effects of hippocampal or prefrontal cortical lesions on three versions of delayed non-matching-to-sample based on positional or spatial cues. Behav. Brain Res. 109: 69-81.

Pothuizen, H.H., Zhang, W.N., Jongen-Relo, A.L., Feldon, J., and Yee, B.K. 2004. Dissociation of function between the dorsal and the ventral hippocampus in spatial learning abilities of the rat: A within-subject, within-task comparison of reference and working spatial memory. Eur. J. Neurosci. 19: 705-712.

Poucet, B. 1997. Searching for spatial unit firing in the prelimbic area of the rat medial prefrontal cortex. Behav. Brain Res. 84: 151-159.

Schoenbaum, G., Setlow, B., and Ramus, S.J. 2003. A systems approach to orbitofrontal cortex function: Recordings in rat orbitofrontal cortex reveal interactions with different learning systems. Behav. Brain Res. 146: 19-29.

Scoville, W.B. and Milner, B. 1957. Loss of recent memory after bilateral hippocampal lesions. J. Neurol. Neurosurg. Psychiatry 20: 11-21.

Seamans, J.K. and Phillips, A.G. 1994. Selective memory impairments produced by transient lidocaine-induced lesions of the nucleus accumbens in rats. Behav. Neurosci. 108: 456-468.

Seamans, J.K., Floresco, S.B., and Phillips, A.G. 1995. Functional differences between the prelimbic and anterior cingulate regions of the rat prefrontal cortex. Behav. Neurosci. 109: 1063-1073.

Siapas, A.G., Lubenov, E.V., and Wilson, M.A. 2005. Prefrontal phase locking to hippocampal theta oscillations. Neuron 46: 141-151.

Sloan, H.L., Good, M., and Dunnett, S.B. 2006. Double dissociation between hippocampal and prefrontal lesions on an operant delayed matching task and a water maze reference memory task. Behav. Brain Res. 171: 116-126.

Squire, L.R. 1987. Memory and brain. Oxford University Press, New York. Stuss, D.T. and Alexander, M.P. 2000. Executive functions and the frontal lobes: A conceptual view. Psychol. Res. 63: 289-298.

Thierry, A.M., Gioanni, Y., Degenetais, E., and Glowinski, J. 2000. Hippocampo-prefrontal cortex pathway: Anatomical and electrophysiological characteristics. Hippocampus 10: 411-419.

Wang, G.W. and Cai, J.X. 2006. Disconnection of the hippocampal-prefrontal cortical circuits impairs spatial working memory performance in rats. Behav. Brain Res. 175: 329-336.

Whishaw, I.Q. and Tomie, J.A. 1997. Perseveration on place reversals in spatial swimming pool tasks: Further evidence for place learning in hippocampal rats. Hippocampus 7: 361-370.

Wilensky, A.E., Schafe, G.E., and LeDoux, J.E. 1999. Functional inactivation of the amygdala before but not after auditory fear conditioning prevents memory formation. J. Neurosci. 19: RC48 (1-5).

Winer, B.J., Brown, D.R., and Michels, K.M. 1991. Statistical principles in experimental design. McGraw-Hill, New York.

Zahrt, J., Taylor, J.R., Mathew, R.G., and Arnsten, A.F.T. 1997. Supranormal stimulation of $D_{1}$ dopamine receptors in the rodent prefrontal cortex impairs spatial working memory performance. $J$. Neurosci. 17: 8528-8535.

Received November 19, 2007; accepted in revised form December 14, 2007. 


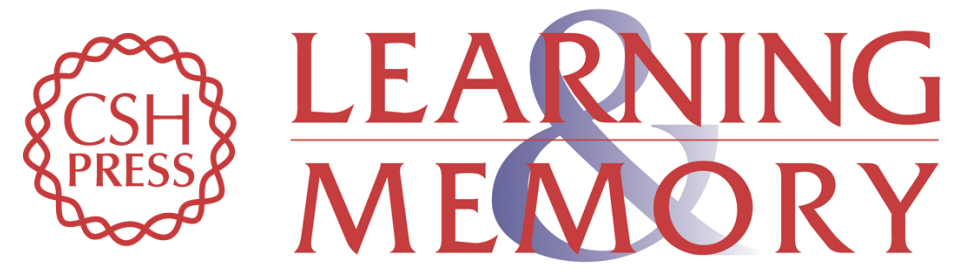

\section{Prefrontal cortex and hippocampus subserve different components of working memory in rats}

Taejib Yoon, Jeffrey Okada, Min W. Jung, et al.

Learn. Mem. 2008, 15:

Access the most recent version at doi:10.1101//m.850808

References This article cites 65 articles, 16 of which can be accessed free at: http://learnmem.cshlp.org/content/15/3/97.full.html\#ref-list-1

License

Email Alerting Receive free email alerts when new articles cite this article - sign up in the box at the Service top right corner of the article or click here. 